Article

\title{
Letnikov vs. Marchaud: A Survey on Two Prominent Constructions of Fractional Derivatives
}

\section{Sergei Rogosin * and Maryna Dubatovskaya}

Department of Economics, Belarusian State University, 4, Nezavisimosti ave, 220030 Minsk, Belarus;

dubatovska@bsu.by

* Correspondence: rogosinsv@gmail.com; Tel.: +375-17-220-22-84

Received: 22 November 2017; Accepted: 20 December 2017; Published: 25 December 2017

Abstract: In this survey paper, we analyze two constructions of fractional derivatives proposed by Aleksey Letnikov (1837-1888) and by André Marchaud (1887-1973), respectively. These derivatives play very important roles in Fractional Calculus and its applications.

Keywords: fractional integrals and derivatives; Grünwald-Letnikov approach; Marchaud approach; fractional differences; Hadamard finite part

MSC: primary 26A33; secondary 34A08; 34K37; 35R11; 39A70

\section{Introduction}

The aim of the paper is to present the essence of two important approaches in Fractional Calculus, namely, those developed by Letnikov (or by Grünwald-Letnikov) and by Marchaud. We collect here the most important results for the corresponding fractional derivatives, compare these constructions and highlight their role in Fractional Calculus and its applications.

In his master thesis (see [1], p. 37) Letnikov (When the thesiswas ready for defence, Letnikov discovered the paper by Grünwald [2] in which the same approach was realised.) defined the left-sided fractional derivative on the interval $\left[x_{0}, x\right]$ via the following limit:

$$
y^{\alpha}(x):=\lim _{h \rightarrow+0} \frac{\sum_{k=0}^{n}(-1)^{k}\left(\begin{array}{l}
\alpha \\
k
\end{array}\right) y(x-k h)}{h^{\alpha}},
$$

where $n h=x-x_{0}$. Similarly, the right-sided fractional derivative is defined:

$$
y^{\alpha)}(x):=\lim _{h \rightarrow+0} \frac{\sum_{k=0}^{n}(-1)^{k}\left(\begin{array}{l}
\alpha \\
k
\end{array}\right) y(x+k h)}{h^{\alpha}} .
$$

These definitions can be used at points on semi-axes $\left[x_{0},+\infty\right)\left(\right.$ or $\left.\left(-\infty, x_{0}\right]\right)$ whenever the function $y(x)$ is defined there. In this case, we formally have to replace the finite sum for an infinite one and remove any restriction on the increment $h$.

The aim of the work by Letnikov was to correct the definition of Liouville [3,4], who supposed that his construction of the fractional derivative is a general one. More exactly, Letnikov tried to overcome Liouville's assumption that the general definition of fractional derivative can be applied only to functions represented in the form convergent Dirichlet type series (see Representation (6)). Detailed description of the difficulties that bring such an assumption is given in [5] (see also Section 2.1 below). (In [6], Letnikov transformed his fractional derivative to the form which coincides with the Riemann's formula if one 
removes from the later so called "additional function". Note that at that time Letnikov did not know the work by Riemann since it was published only later in the first edition of Riemann's collected works in 1876 [7].)

The doctoral thesis by Marchaud was published in complete form in the Journal de Mathématiques Pures et Appliquées in 1927 [8] and reprinted in the series “Théses de L'Entre-Deux-Guerres" in 1965 [9]. His main idea was to generalize the Riemann-Liouville approach. Replacing positive parameter $\alpha$ in the Riemann-Liouville fractional integral:

$$
I_{a}^{\alpha}=\frac{1}{\Gamma(\alpha)} \int_{a}^{x} \frac{f(\tau) d \tau}{(x-\tau)^{1-\alpha}}=\frac{1}{\Gamma(\alpha)} \int_{0}^{x-a} t^{\alpha-1} f(x-t) d t
$$

by negative one, he considered the divergent integral:

$$
\frac{1}{\Gamma(-\alpha)} \int_{0}^{x-a} t^{-\alpha-1} f(x-t) d t
$$

In order to regularize this definition, Marchaud made some transformations in the Riemann-Liouville fractional integral.

For arbitrary values of $\alpha, \operatorname{Re} \alpha>0$, the definition of the Marchaud derivative reads ([10], Section 5.6)

$$
\mathbb{D}_{ \pm}^{\alpha} f(x)=-\frac{1}{\Gamma(-\alpha) A_{l}(\alpha)} \int_{0}^{\infty} \frac{\left(\Delta_{ \pm t}^{l} f\right)(x)}{t^{1+\alpha}} d t, \quad l>\operatorname{Re} \alpha>0
$$

where

$$
A_{l}(\alpha)=\sum_{k=0}^{\infty}(-1)^{k-1}\left(\begin{array}{l}
l \\
k
\end{array}\right) k^{\alpha}, \quad\left(\Delta_{ \pm t}^{l} f\right)(x)=\sum_{k=0}^{\infty}(-1)^{k}\left(\begin{array}{l}
l \\
k
\end{array}\right) f(x \mp k t) .
$$

If $0<\alpha<1$, then the left- and right-sided are defined, respectively (see, e.g., [10], Section 5.4),

$$
\mathbb{D}_{+}^{\alpha} f(x)=\frac{\alpha}{\Gamma(1-\alpha)} \int_{0}^{\infty} \frac{f(t)-f(x-t)}{t^{1+\alpha}} d t, \quad \mathbb{D}_{-}^{\alpha} f(x)=\frac{\alpha}{\Gamma(1-\alpha)} \int_{0}^{\infty} \frac{f(t)-f(x+t)}{t^{1+\alpha}} d t .
$$

Since the integral in the right-side formula (3) is in general diverging, then the Marchaud derivative can be defined via the limit of the truncated derivative (if it exists):

$$
\mathbb{D}_{ \pm}^{\alpha} f(x)=\lim _{\varepsilon \rightarrow+0} \mathbb{D}_{ \pm, \varepsilon}^{\alpha} f(x)=\lim _{\varepsilon \rightarrow+0}-\frac{1}{\Gamma(-\alpha) A_{l}(\alpha)} \int_{\varepsilon}^{\infty} \frac{\left(\Delta_{ \pm t}^{l} f\right)(x)}{t^{1+\alpha}} d t
$$

It is seen from (1), (3) that there exists a formal relationship of the Grünwald-Letnikov and Marchaud derivatives. It will be discussed in more detail below. It should be noted that both constructions (by Letnikov and by Marchaud) are applicable to a much wider class of the functions than Liouville's construction (though all of them coincide on "good functions", see [10]).

Nowadays, both derivatives attract more attention among experts from different branches of Science. These derivatives are suitable for numerical analysis of the corresponding fractional models since they are defined based on discretization (see, e.g., [11,12]). Among applications of such derivatives, we have to mention those in different physical models (see, e.g., [13-21] and an extending survey in [22], Chapter 8), as well as in the study of certain problems of operator theory (see, e.g., [23-25]) and results describing interrelation between fractional calculus and fractal geometry (see, e.g., [26,27]). Since the Marchaud derivative is less known than Grünwald-Letnikov, Riemann-Liouville or Caputo derivatives, we have to mention some features of this construction that 
are important in application. First, Marchaud construction allows more freedom for the behavior at infinity (e.g., to the exogene variable $X$ in the accelerator-multiplied model with memory as in [28]). Second, the Marchaud idea to regularize the Liouville fractional derivative by using finite differences met applications at the study of fractional differential equations as in [29]. Third, the Marchaud derivative possesses a simple and straightforward generalization to multi-dimensional case (see [10], Section 5.24), which is useful for models involving fractional powers of operators.

In this article, we analyze the construction and nature of the Grünwald-Letnikov and Marchaud derivatives starting from the original ideas of their creators. Section 2 is devoted to the Letnikov's contribution and Section 3 deals with Marchaud's approach. Some notes on common features and differences between these two constructions are presented in Section 4 .

\section{Letnikov Contribution to Fractional Calculus}

Here, we describe the construction of so-called "general differentiation" (The words utilized by Letnikov for his derivative.) (or differentiation of arbitrary order) proposed by Alexej Vasil'evich Letnikov in the 1860s. It is based on certain results by Liouville that Letnikov considered as the most important results on fractional differentiation.

\subsection{Preliminaries}

Let us briefly describe some results of predecessors to the work by Letnikov. In spite of the fact that the idea of fractional derivative goes back to the end of the 17th century (see [30]), the real results in the area were made by Liouville [3,4].

Liouville applied his construction to the function representing in the form of the following (convergent!) series:

$$
y(x)=\sum_{k=1}^{\infty} A_{k} e^{m_{k} x}
$$

For such functions, he used Leibnitz's idea of an arbitrary order differentiation of the exponential function:

$$
\frac{d^{p} y}{d x^{p}}:=\sum_{k=1}^{\infty} A_{k} m_{k}^{p} e^{m_{k} x}
$$

Liouville considered definition (7) as only possible, stressing (see [1], p. 14): “...it is impossible to get an exact and complete understanding of the nature of the arbitrary order derivative without taken certain series representation of the function".

Letnikov noted that Liouville's construction being deeply justified has an essential drawback. It follows from the definition (7) that it can be used only for functions whose derivatives (of all positive integer orders) are vanishing at infinity. Liouville himself met the first difficulty trying to apply his definition to power function with positive exponent $x^{m}, m>0$, which does not satisfy the above condition. To overcome this difficulty he started with the function $y(x)=\frac{1}{x^{m}}, m>0$. It follows from the definition of the $\Gamma$-function that the following relation holds:

$$
\frac{1}{x^{m}}=\frac{1}{\Gamma(m)} \int_{0}^{\infty} e^{-z x} z^{m-1} d z
$$

The right-hand side of this formula can be considered as an expansion of the type (6), namely, $\sum A_{n} e^{-n x}$ with $A_{n}$ being sufficiently small as $x \rightarrow \infty$. Thus, formula (7) applied in this case leads to the following result:

$$
\frac{d^{p} \frac{1}{x^{m}}}{d x^{p}}=\frac{1}{\Gamma(m)} \int_{0}^{\infty} e^{-z x}(-z)^{p} z^{m-1} d z=\frac{(-1)^{p}}{\Gamma(m) x^{m+p}} \int_{0}^{\infty} e^{-t} t^{p+m-1} d z=\frac{(-1)^{p} \Gamma(m+p)}{\Gamma(m) x^{m+p}},
$$


which coincides with the celebrated Euler formula for the arbitrary order derivative of the power function.

Note that Liouville considered only the case when $m>0$ and $m+p>0$. (At this time, the Legendre-Gauss definition of $\Gamma$-function of the complex argument was not known.) For the remaining cases, he used the notion of so called additional functions in order to correct the above definition. These are the functions whose derivative of order $(-p)$ is equal to zero. Liouville gave a proof that additional functions should have the form:

$$
A_{0}+A_{1} x+\ldots+A_{n} x^{n},
$$

with certain finite power $n$ and arbitrary constant coefficients $A_{j}$. (This proof was not considered satisfactory by many mathematicians even in Liouville's time.)

During the next 30 years, several attempts to correct Liouville's construction were made (the most known results were due to Peacock, Kelland, Tardi, Roberts, see ([1], pp. 16-23), see also Chapter 1 by Butzer and Westphal in [18]). Anyway, only in 1867-1868, it was proposed by A.K. Grünwald [2] and by A.V. Letnikov [5] a really general definition of the fractional derivative. Both constructions (which differ only in few details) are based on the following formula for representation of the derivative of an arbitrary positive integer order via finite differences:

$$
\frac{d^{p} f(x)}{d x^{p}}=\lim _{h \rightarrow+0} \frac{f(x)-\left(\begin{array}{l}
p \\
1
\end{array}\right) f(x-h)+\left(\begin{array}{l}
p \\
2
\end{array}\right) f(x-2 h)-\ldots+(-1)^{n}\left(\begin{array}{l}
p \\
n
\end{array}\right) f(x-n h)}{h^{p}},
$$

where $n$ is an arbitrary positive number, $n \geq p$.

\subsection{Letnikov's Construction}

In his construction [5] (see also [1] where the article [5] is reprinted practically without any changes.), Letnikov started with the now known formula (9) of the derivative of an integer order $p=m$. By assuming $p$ being an arbitrary positive number and $n$ arbitrary sufficiently large positive integer number, he supposed by definition:

$$
f^{p)}(x):=\lim _{h \rightarrow 0} \frac{f(x)-\left(\begin{array}{c}
p \\
1
\end{array}\right) f(x-h)+\left(\begin{array}{l}
p \\
2
\end{array}\right) f(x-2 h)-\ldots+(-1)^{n}\left(\begin{array}{l}
p \\
n
\end{array}\right) f(x-n h)}{h^{p}},
$$

where $\left(\begin{array}{c}k \\ r\end{array}\right)=\frac{k(k-1) \ldots(k-r+1)}{1 \cdot 2 \cdot \ldots \cdot r}$. It was noted that if $p$ is a positive integer, $p \leq n$, then formula (10) leads to the derivative of $p$-th order.

Letnikov also considered an expression:

$$
f^{-p)}(x):=\lim _{h \rightarrow 0} h^{p}\left[f(x)+\left(\begin{array}{l}
p \\
1
\end{array}\right) f(x-h)+\left(\begin{array}{l}
p \\
2
\end{array}\right) f(x-2 h)+\ldots+\left(\begin{array}{l}
p \\
n
\end{array}\right) f(x-n h)\right] .
$$

If $p$ is a positive integer in the last formula, then $f^{-p)}(x)$ is vanishing whenever $n$ is finite. Hence, it is interesting to consider the case when $n$ tends to infinity as $h \rightarrow 0$. Assuming $h=\left(x-x_{0}\right) / n$ Letnikov defined the following object (called the derivative of a negative order in finite limits by him)

$$
\left[D^{-p} f(x)\right]_{x_{0}}^{x}:=\lim _{h \rightarrow 0} f^{-p)}(x) .
$$

It was shown that, for $p$ being a positive integer, the following relation holds whenever the integral on the right-hand side exists: 


$$
\left[D^{-p} f(x)\right]_{x_{0}}^{x}=\lim _{h \rightarrow 0} \sum_{r=0}^{n} h^{p}\left(\begin{array}{c}
p \\
r
\end{array}\right) f(x-r h)=\frac{1}{1 \cdot 2 \cdot \ldots \cdot(p-1)} \int_{x_{0}}^{x}(x-\tau)^{p-1} f(\tau) d \tau .
$$

It follows from (12) that if this formula is valid for certain positive integer $p$, then it is valid for $p+1$ too. Moreover, the above introduced object $\left[D^{-p} f(x)\right]_{x_{0}}^{x}$ means the function whose $p$-th derivative coincides with $f(x)$ :

$$
\frac{d^{p}}{d x^{p}}\left[D^{-p} f(x)\right]_{x_{0}}^{x}=f(x)
$$

and

$$
\frac{d^{j}}{d x^{j}}\left[D^{-p} f(x)\right]_{\left.x_{0}\right|_{x=x_{0}} ^{x}}^{x}=0, \quad \forall j=0,1, \ldots, p-1 .
$$

The above definition and its properties constitute the base for further generalizations. Thus, by using the properties of binomial coefficients, Letnikov proved that for any function $f$ continuous on $\left[x_{0}, x\right]$ there exist the following limits:

$$
\lim _{n \rightarrow \infty} \frac{\sum_{r=0}^{n}(-1)^{r}\left(\begin{array}{l}
p \\
r
\end{array}\right) f(x-r h)}{h^{p}}, \lim _{n \rightarrow \infty} \sum_{r=0}^{n} h^{p}\left(\begin{array}{l}
p \\
r
\end{array}\right) f(x-r h),
$$

where $h=\left(x-x_{0}\right) / n$ and $p \in \mathbb{C}, \operatorname{Re} p>0$. The values of these limits denoted by him $\left[D^{p} f(x)\right]_{x_{0}}^{x}$ and $\left[D^{-p} f(x)\right]_{x_{0}}^{x}$, respectively, are formally equal in this case to:

$$
\begin{aligned}
& {\left[D^{p} f(x)\right]_{x_{0}}^{x}=\frac{1}{\Gamma(-p)} \int_{x_{0}}^{x} \frac{f(\tau) d \tau}{(x-\tau)^{p+1}},} \\
& {\left[D^{-p} f(x)\right]_{x_{0}}^{x}=\frac{1}{\Gamma(p)} \int_{x_{0}}^{x} \frac{f(\tau) d \tau}{(x-\tau)^{1-p}} .}
\end{aligned}
$$

Formula (13) gives the derivative of arbitrary order $p \in \mathbb{C}, \operatorname{Re} p>0$, and formula (14) gives the integral of arbitrary order $p \in \mathbb{C}, \operatorname{Re} p>0$. As it was already mentioned, the integral in (14) exists whenever $f$ is continuous, though it is not the case for the integral in (13). To overcome this difficulty, Letnikov transformed the right-hand side of (13) to the form:

$$
\left[D^{p} f(x)\right]_{x_{0}}^{x}=\sum_{k=0}^{m} \frac{f^{(k)}\left(x_{0}\right)\left(x-x_{0}\right)^{-p+k}}{\Gamma(-p+k+1)}+\frac{1}{\Gamma(-p+m+1)} \int_{x_{0}}^{x} \frac{f^{(m+1)}(\tau) d \tau}{(x-\tau)^{p-m}} .
$$

Surely, an assumption of existence and continuity of all derivatives up to the order $m+1$ is sufficient for this representation. It is suitable here to take $m=[\operatorname{Re} p]$.

Integration by parts in (14) leads to an analogous formula for $\left[D^{-p} f(x)\right]_{x_{0}}^{x}$, which is valid under the same conditions for any integer positive $m$ :

$$
\left[D^{-p} f(x)\right]_{x_{0}}^{x}=\sum_{k=0}^{m} \frac{f^{(k)}\left(x_{0}\right)\left(x-x_{0}\right)^{p+k}}{\Gamma(p+k+1)}+\frac{1}{\Gamma(p+m+1)} \int_{x_{0}}^{x} \frac{f^{(m+1)}(\tau) d \tau}{(x-\tau)^{p-1+m}} .
$$

It can be considered also a special case when $x_{0} \rightarrow+\infty$. It follows from (14):

$$
\left[D^{-p} f(x)\right]_{+\infty}^{x}=\frac{(-1)^{p}}{\Gamma(p)} \int_{0}^{+\infty} \tau^{p-1} f(x+\tau) d \tau,
$$


and from (15) we obtain:

$$
\left[D^{p} f(x)\right]_{+\infty}^{x}=\frac{(-1)^{m+1-p}}{\Gamma(-p+m+1)} \int_{0}^{+\infty} \tau^{m-p} f^{(m+1)}(x+\tau) d \tau .
$$

Letnikov noted that the integrals on the right-hand side of (18) and (17) converge in particular if $\lim _{x_{0} \rightarrow+\infty} f^{(k)}\left(x_{0}\right)=0, k=0, \ldots, m$. This is exactly the class of functions considered by Liouville and formulas (17) and (18) that coincide with the corresponding formulas presented in [3,4].

\subsection{Further Results on Grünwald-Letnikov Fractional Derivative/Integral}

After the death of Letnikov, his theory did not get a serious development neither in Russia nor abroad. We have to mention the work by Nekrasov [31] who tried to overcome additional assumptions by Letnikov ensuring existence of the integrals appeared after passing to a limit. In his work, Nekrasov used the technique of Complex Analysis, in particular, the behaviour of complex valued function in a neighbourhood of branch-points.

Only much later, the Grünwald-Letnikov approach was retranslated into the modern language. The most essential improvement was presented in the article by Butzer and Westphal [32] and by Bugrov [33]. Historical remarks describing this development is presented in ([10], §23). Here we present the most essential results on the Grünwald-Letnikov fractional derivative.

In [10], the Grünwald-Letnikov fractional derivative is defined via differences of arbitrary order for functions from the space $X=X\left(\mathbb{R}^{1}\right)$ (it is either $L_{p}\left(\mathbb{R}^{1}\right)$ or $\mathcal{C}\left(\mathbb{R}^{1}\right)$ ) or for $2 \pi$-periodic functions from the same type of spaces denoting $X_{2 \pi}=X(0,2 \pi)$. Such differences of arbitrary order are defined as follows:

$$
\left(\Delta_{ \pm t}^{\alpha} f\right)(x)=\sum_{k=0}^{\infty}(-1)^{k}\left(\begin{array}{c}
\alpha \\
k
\end{array}\right) f(x \mp k t) .
$$

Left-sided/right-sided differences correspond to upper/lower signs. Letnikov proved convergence of differences (19) for any $\alpha>0$ and each bounded continuous function. In fact, absolute and uniform convergence follows already for any bounded function. If the function $f$ belongs to one of the above spaces, then the convergence takes place in the norm of the corresponding space. Classical definition of the Grüwald-Letnikov fractional derivative remains the same as in original papers [2,5]:

$$
f_{ \pm}^{\alpha}(x)=\lim _{h \rightarrow+0} \frac{\left(\Delta_{ \pm t}^{\alpha} f\right)(x)}{h^{\alpha}}, \quad \alpha>0
$$

If the convergence in (20) is understood in the norm of the corresponding space, then $f_{ \pm}^{\alpha)}(x)$ is called the strong Grüwald-Letnikov fractional derivative.

Existence of the strong Grüwald-Letnikov fractional derivative in the periodic case gives the following:

Theorem 1. (Theorem 20.1 [10]) Let $f \in X_{2 \pi}$. The strong Grüwald-Letnikov fractional derivative exists in the corresponding space iff there exists a function $\varphi_{ \pm} \in X_{2 \pi}$ such that the following representation holds

$$
f(x)=I_{ \pm}^{(\alpha)} \varphi_{ \pm}+f_{0}, \quad f_{0}=\frac{1}{2 \pi} \int_{0}^{2 \pi} f(x) d x
$$

where $I_{ \pm}^{(\alpha)}$ are the Liouville fractional integrals:

$$
I_{+}^{(\alpha)} \phi(x)=\frac{1}{\Gamma(\alpha)} \int_{-\infty}^{x} \frac{\phi(\tau) d \tau}{(x-\tau)^{1-\alpha}}, \quad I_{-}^{(\alpha)} \phi(x)=\frac{1}{\Gamma(\alpha)} \int_{x}^{+\infty} \frac{\phi(\tau) d \tau}{(\tau-x)^{1-\alpha}} .
$$




$$
\text { If } f_{ \pm}^{\alpha)}(x) \text { exists, then } \varphi_{ \pm}(x)=f_{ \pm}^{\alpha)}(x)
$$

In a nonperiodic case, an existence (in sense of $L_{p}$-norm, $p>1$ ) of the strong Grünwald-Letnikov fractional derivative of the function $f \in L_{r}\left(\mathbb{R}^{1}\right)$ implies that $f$ can be represented by the (Liouville) fractional integral of this fractional derivative.

\subsection{Short Biography of A.V. Letnikov}

Alexey Vasil'evich Letnikov was born in Moscow on 1 January 1837 in the family of a rather poor nobleman. His father died when Alexey was 8 years old. His mother tried to give an education to Alexey and his sister. His mother sent Alexey to grammar school in 1847. In spite of his evident abilities, he was not too successful in education. Therefore, he was moved to Konstantin's land surveyors institute (full-time provisional military type institute). This was a second rank educational establishment. Anyway, the director of the Institute tried to do the best for good students. He discovered Alexey's big interest in mathematics and supported his growth in the subject. The director decided to prepare him for a career of a teacher in mathematics in Konstantin's land surveyors institute. To get the corresponding position, Letnikov was sent to Moscow University and studied mathematics there for two years (1856-1858) as an extern student. After graduation, he was sent to Paris in order to extend his knowledge at the most well-known mathematical center for two years and to study the structure and the content of the technical education in France.

In Paris, Letnikov attended the lectures of many well-known mathematicians (Liouville among them) in the Ecole Polytechnique, College de France and Sorbonne. Returning from Paris in December 1860, he was appointed as a teacher in the engineering class of Konstantin's land surveyors institute and started to teach Probability Theory. Letnikov actively participated in mathematical life in Moscow. In particular, he was among the founders of Moscow Mathematical Society in 1864.

In 1863, a new Statute of Higher Education was approved. Among other regulations, it was supposed to enlarge a number of chairs at universities and to recruit new university teachers. To get a position in university, one had either to pass the graduation gymnasium's exams or to receive the degree at a foreign university. Letnikov decided to use the second option. In 1867, he defended a Ph.D. "Über die Bedingungen der Integrabilitát einiger Differential-Gleichungen" at Leipzig university.

In 1868, Konstantin's land surveyors institute moved from the military ministry to a civilian one and its teachers had to leave the military service. Therefore, Letnikov searched for another position as a teacher of mathematics. At that time, the Imperial Technical College (now Bauman's Technical University) was reopened after being transformed from the Moscow Industrial School and Letnikov got a position there. He was working at this College up to 1883 when he moved to Alexandrov's Commercial College sharing this job with a part-time teacher at Konstantin's land surveyors institute and at the Imperial Technical College. It was an active time for him, and he was awarded the degree of a state councillor, received the order of Saint Stanislav and was appointed in 1884 as a corresponding member of St.-Petersburg Academy of Sciences (by recommendation of Imshenetsky, Bunyakovsky and Backlund).

Letnikov's main scientific results were obtained during the 20 years starting from the middle of the 1860s. In 1866, he began to work on the theme "differentiation of arbitrary order". When the work was almost ready (and he supposed to defend it as a Master's thesis), he found the article by a professor from Prague university, A.K. Grünwald [2], who exploited the same idea. Only due to a professor Davidov from Moscow University (who knew the research of Letnikov and Grünwald and recommended to Letnikov to complete the presentation) was the Master's thesis defended and published at Mathematical Sbornik in 1868 [5]. (This work as well as the work [34] was reprinted in [1].) Letnikov's theory was not completely recognized at that time. Thus, in 1972, Sonin (future academician) published a paper [35] in which he criticized some statements of Letnikov's theory. Letnikov answered [34] in the same year with an explanation of the basic results of his theory recovering mistakes made by Sonin. In 1874, Letnikov published a paper [6] in which he corrected the models taken 
by Liouville in his works [3] and showed how one can use the new theory of fractional differentiation to attack the problems arising. It should be noted that Letnikov published all his results in Russian at Mathematical Sbornik. This is why these works are not too familiar for mathematicians outside Russia.

The personal life of A.V. Letnikov was quite heavy. He married in 1863 and had a large family. Because of that, he had to work hard teaching at several institutions. At the end of the 1880s, Letnikov should have received a state pension and was supposed to leave teaching and concentrate on research. He was dreaming of getting a position at Moscow University, but it did not happen, since, at the opening ceremony of a new building of Alexandrov's Commercial College, he caught a cold. He had no serious illness before and continued to deliver lectures. However, this time his disease was serious. Moreover, it was complicated by typhoid fever and he died on 27 February 1988.

\section{Marchaud Contribution to Fractional Calculus}

\subsection{Main Constructions}

In his thesis (published in 1927 [8] and reprinted in [9]), André Paul Marchaud (1887-1973) started from the Riemann-Liouville construction and tried to generalize it by using new knowledge on the properties of the functions of real variables. By examination of the main properties of the Riemann-Lioville integral $\left(I_{a+}^{(\alpha)} f\right)(x)=\frac{1}{\alpha} \int_{a}^{x-a} \tau^{\alpha-1} f(x-\tau) d \tau$ and derivative $\left(D_{a+}^{(\alpha)} f\right)(x)=$ $\frac{d^{n}}{d x^{n}} I_{a+}^{n-\alpha} f(x), n>\alpha$, he noted that $\left(I_{a+}^{(\alpha)} f\right)$ is well-defined for any bounded function (respectively, bounded and integrable in the case of Liouville fractional integral with integration along semi-axes). As for the Riemann-Liouville fractional derivative, it can be unbounded at the lower end of integration as it follows, e.g., from the simple example:

$$
\left(D_{a+}^{(\alpha)}(1)\right)(x)=\frac{(x-a)^{-\alpha}}{\Gamma(1-\alpha)}
$$

Marchaud studied such situation, taking into account the following consideration. If the function $f$ admits a bounded derivative of an integer order $r$ on the interval $\left(a, a_{1}\right)$, then there exists the derivative $\left(D_{a+}^{\left(\alpha^{\prime}\right)}(f)\right)(x)$ of any order $\alpha^{\prime}<r$. Such derivatives can be defined via formula:

$$
\left(D_{a+}^{\left(\alpha^{\prime}\right)}(f)\right)(x)=\left(I_{a+}^{\left(r-\alpha^{\prime}\right)} f^{(r)}\right)(x)+\sum_{j=0}^{r-1} \frac{(x-a)^{j-\alpha^{\prime}}}{\Gamma\left(j+1-\alpha^{\prime}\right)} f^{(j)}(x) .
$$

Any derivative $D_{a+}^{\left(\alpha^{\prime}\right)}(f)$ is unbounded at $x=a$, but it is bounded on each subinterval $\left(a^{\prime}, a_{1}\right) \subset\left(a, a_{1}\right)$.

Marchaud proved the following relation (valid in the above conditions with noninteger $\alpha$ and $r=[\alpha]$ )

$$
f(x)=\left(I_{a^{\prime}+}^{(\alpha)} D_{a+}^{(\alpha)} f\right)(x)+\sum_{j=1}^{r} \frac{\left(x-a^{\prime}\right)^{j+\alpha-r-1}}{\Gamma(j+\alpha-r)} F^{(j)}\left(a^{\prime}\right)+\frac{\left(x-a^{\prime}\right)^{\alpha-r}}{\Gamma(\alpha-r) \Gamma(r+1-\alpha)} \int_{a}^{a^{\prime}} \frac{\left(a^{\prime}-t\right)^{r-\alpha} f(t) d t}{(x-t)}
$$

where

$$
F(x):=\left(I_{a+}^{(r+1-\alpha)} f\right)(x)
$$

It gives, in particular, that if all derivatives $\left(D_{a^{\prime}+}^{\left(\alpha^{\prime}\right)} f\right)(x)\left(\alpha^{\prime}<\alpha\right)$ exist and are continuous on the interval $\left(a^{\prime}, a_{1}\right)$, then there exists the derivative $\left(D_{a+}^{(\alpha)} f\right)(x)$, which is bounded on $\left(a^{\prime}, a_{1}\right)$. Such result was obtained by Montel (see [36]) under additional conditions that $\left(D_{a+}^{(\alpha)} f\right)(x)$ is bounded on the interval $\left(a, a_{1}\right)$. Montel proved: 


$$
\left(D_{a+}^{\left(\alpha^{\prime}\right)} f\right)(x)=\left(I_{a+}^{\left(\alpha-\alpha^{\prime}\right)} D_{a+}^{(\alpha)} f\right)(x)+\sum_{j=1}^{r} \frac{(x-a)^{j+\alpha-\alpha^{\prime}-r-1}}{\Gamma\left(j+\alpha-\alpha^{\prime}-r\right)} F^{(j)}(a), \quad \alpha^{\prime}<\alpha
$$

Thus, sufficient conditions for continuity of these derivatives at $x=a$ is vanishing of all derivatives $F^{(j)}(a)$ and $\lim _{x \rightarrow a}(x-a)^{-\alpha+1} f(x)=0$.

Marchaud noted that relation (23) can not be taken for a definition of the derivative of a noninteger order since the function $f$ remains in the last term on the right-hand side. Therefore, he tried to use another approach to the definition taking into account the above consideration on the behaviour of the function at the initial point $x=a$. The starting point for this definition was a formula obtained by the formal replacement of positive $\alpha$ in the definition of fractional integral for a negative parameter:

$$
\left(D_{a+}^{(\alpha)} f\right)(x)=\frac{1}{\Gamma(-\alpha)} \int_{0}^{x-a} \frac{f(x-t) d t}{t^{\alpha+1}}
$$

Since the integral on the right-hand side is divergent, this definition needs some transformation. For the function defined on the interval $\left(a, a_{1}\right)$, it was proposed to extend it by zero on $(-\infty, a]$ and to denote the corresponding fractional integral by $f_{(\alpha)}$ and fractional derivative by $f^{(\alpha)}$.

In order to find a proper transformation, Marchaud started to regularize fractional integrals. In the following formula (valid, e.g., for exponential function):

$$
f_{(\alpha)}(x) \int_{0}^{\infty} t^{\alpha-1} e^{-t} d t=\int_{0}^{\infty} t^{\alpha-1} f(x-t) d t
$$

he replaced $t$ for $k_{j} t(j=0,1, \ldots, p)$ and made a linear combination of the obtained equalities with unknown coefficients $C_{j}, j=0,1, \ldots, p$. It leads to the following formula:

$$
f_{(\alpha)}(x) \int_{0}^{\infty} t^{\alpha-1} \psi(t) d t=\int_{0}^{\infty} t^{\alpha-1} \varphi(x, t) d t
$$

where

$$
\psi(t)=\sum_{j=0}^{p} C_{j} e^{-k_{j} t}, \quad \varphi(x, t)=\sum_{j=0}^{p} C_{j} f\left(x-k_{j} t\right) .
$$

Then, replacing $\alpha$ for $-\alpha$, we arrive at the following formal relation, which needs some extra conditions for its validity:

$$
f^{(\alpha)}(x) \int_{0}^{\infty} t^{-\alpha-1} \psi(t) d t=\int_{0}^{\infty} t^{-\alpha-1} \varphi(x, t) d t .
$$

If the integral:

$$
\gamma(\alpha)=\int_{0}^{\infty} t^{-\alpha-1} \psi(t) d t
$$

has a sense for certain $\alpha=\alpha_{0}$, then $\gamma_{\alpha}$ is defined and continuous for all $\alpha \leq \alpha_{0}$.

Denoting $r=[\alpha]$, one can see that $\gamma(\alpha)$ has a sense whenever $\psi(t)$ has the order of infinitesimality equal to $r+1$. It is required:

$$
\sum_{j=0}^{p} k_{j}^{s} C_{j}=0, \quad s=0,1, \ldots, r
$$


$r$-times integration by parts gives:

$$
\gamma(\alpha)=\Gamma(-\alpha) \sum_{j=0}^{p} k_{j}^{\alpha} C_{j} \quad(\alpha \neq r) ; \quad \gamma(r)=\lim _{\alpha \rightarrow r} \gamma(\alpha)=\frac{(-1)^{r+1}}{r !} \sum_{j=0}^{p} k_{j}^{r} C_{j} \log k_{j} .
$$

The most simple function $\psi(t)$ that has an order of infinitesimality equal to $p$ is the following one:

$$
\psi(t)=e^{-t}\left(1-e^{-t}\right)^{p}=e^{-t}-\left(\begin{array}{c}
p \\
1
\end{array}\right) e^{-2 t}+\left(\begin{array}{l}
p \\
2
\end{array}\right) e^{-3 t}+\ldots
$$

In this case:

$$
\varphi(x, t)=f(x-t)-\left(\begin{array}{c}
p \\
1
\end{array}\right) f(x-2 t)+\left(\begin{array}{l}
p \\
2
\end{array}\right) f(x-3 t)+\ldots
$$

The simple properties of the Marchaud derivatives are similar to that of the Liouville derivative, e.g., permutability with the operators of reflection, translation and scaling (see [10], Section 2.5), composition formulas with the singular integral operator $S$ and relation between $\mathbb{D}_{-}^{\alpha}$ and $\mathbb{D}_{+}^{\alpha}$ :

$$
\left(\mathbb{D}_{-}^{\alpha} f\right)=\cos \alpha \pi\left(\mathbb{D}_{+}^{\alpha} f\right)-\sin \alpha \pi\left(S \mathbb{D}_{+}^{\alpha} f\right) .
$$

Among characteristic properties, we have to point out the vanishing of the Marchaud derivative on the constant function:

$$
\mathbb{D}_{ \pm}^{\alpha} \text { const } \equiv 0
$$

For all $\alpha>0$, the Marchaud derivative $\mathbb{D}_{ \pm}^{\alpha}$ is defined on all bounded functions $f \in \mathcal{C}^{[\alpha]}\left(\mathbb{R}^{1}\right)$ satisfying the following condition:

$$
\left|f^{([\alpha])}(x+h)-f^{([\alpha])}(x)\right| \leq A(x)|h|^{\lambda} .
$$

\subsection{Condition on Convergence}

In the case of "small" parameter $\alpha$ (see (4)), the Marchaud derivative is defined, for instance, in the class of the locally Hölder continuous bounded functions $H_{\text {loc }}^{\lambda}(\mathbb{R})$ with Hölder exponent $\lambda>\alpha$. Further analysis of convergence was done by Marchaud himself (see [8], Chapter 2). He proposed to introduce the fractional derivative $f^{(\alpha)}$ by formula (27) (with a proper choice of the constant $\gamma(\alpha)$ ). He gave the following condition for the existence of such derivative: the necessary and sufficient condition for a function $f$ continuous on $\left(a, a_{1}\right)$ to admit the fractional derivative $D_{a+}^{\alpha^{\prime}} f$ of any order $\alpha^{\prime} \leq \alpha$ is the uniform convergence of the integral $\int_{\varepsilon}^{\infty} t^{-\alpha-1} \varphi(x, t) d t$ on any subinterval $\left(a^{\prime}, a_{1}\right) \subset\left(a, a_{1}\right)$.

It was noted that the order of infinitesimality of:

$$
\left(\Delta_{h}^{n} f\right)(x):=f(x+n h)-\left(\begin{array}{c}
n \\
1
\end{array}\right) f(x+(n-1) h)+\left(\begin{array}{l}
n \\
2
\end{array}\right) f(x+(n-2) h)+\ldots
$$

depends on the behaviour of the function $f$ at a point $x$. For the function $f$ defined and bounded on $(0,1)$, Marchaud (following [37]) considered the properties of the generalized modulus of continuity defined as:

$$
\omega_{n}(\delta)=\omega_{n}(\delta ; f(x)):=\sup _{|h| \leq \delta \leq \frac{1}{n}}\left(\Delta_{h}^{n} f\right)(x) \quad(0 \leq x \leq 1,0 \leq x+n h \leq 1)
$$


Then, the following statement is valid: letting the integral $\int_{0} t^{-\alpha-1} \omega_{n}(t) d t$ be convergent for certain $\alpha$ and $n(\alpha<n)$, then the function $f$ admits the derivative of any order $\leq \alpha$ given by formula (27).

For instance,

$$
f^{\prime}(x)=\frac{1}{2 \log 2} \int_{0}^{\infty} t^{-2}[f(x)-2 f(x-t)+f(x+2 t)] d t
$$

and, for $n=1,0<\alpha<1$,

$$
f^{(\alpha)}=\frac{\alpha}{\Gamma(1-\alpha)} \int_{0}^{\infty} t^{-\alpha-1}[f(x)-f(x-t)] d t .
$$

Finally, Marchaud made the following remark ([8], p. 396) concerning the differences of noninteger order (citing the paper by Liouville):

$$
\left(\Delta_{h}^{\alpha} f\right)(x):=f(x+\alpha h)-\left(\begin{array}{c}
\alpha \\
1
\end{array}\right) f(x+(\alpha-1) h)+\left(\begin{array}{c}
\alpha \\
2
\end{array}\right) f(x+(\alpha-2) h)+\ldots
$$

He proved that if the following limit exists:

$$
\lim _{\delta \rightarrow+0} \delta^{-\alpha} \Delta_{\delta}^{\alpha} f(x)=g(x)
$$

for certain $\alpha>0$, then the following relation holds (Thus, $g(x)$ can be considered as a fractional derivative of the function $f(x)$ of order $\alpha)$ :

$$
f(x)=I^{(\alpha)} g(x)
$$

\subsection{Further Results on the Marchaud Derivative}

As it was already mentioned, the Marchaud derivative appeared due to a formal replacement of the positive parameter $\alpha$ for negative one $-\alpha$ in the definition of the Liouville fractional integral. The appeared object is not well-defined. In order to give a sense to the integral in (3), one can use Hadamard's finite part (p.f.) (see [10], Lemma 5.2):

$$
\left(\mathbb{D}_{ \pm}^{\alpha} f\right)(x)=p \cdot f \cdot\left(I_{ \pm}^{(-\alpha)} f\right)(x)
$$

An application of this approach gives, in particular, the following result (see [10], Lemma 5.3): let $f \in C_{\text {loc }}^{m, \lambda}(\mathbb{R}), 0 \leq \lambda<1, m=[\alpha], \alpha>0, \alpha \neq 1,2, \ldots$. Then, the following representation holds:

$$
p . f . \int_{0}^{\infty} \frac{f(x-t) d t}{t^{1+\alpha}}=\int_{0}^{\infty} \frac{f(x-t)-\sum_{k=0}^{m} \frac{(-1)^{k} f^{(k)}(x)}{k !}}{t^{1+\alpha}} d t .
$$

The Marchaud derivative is a suitable object for representation of the fractional powers of the operators. Thus, for instance,

$$
\left(\frac{d}{d x}\right)^{\alpha}=\frac{1}{\Gamma(-\alpha)} \int_{0}^{\infty} \frac{\phi(x-t)-\phi(x)}{t^{1+\alpha}} d t=\left(\mathbb{D}_{+}^{(\alpha)} \phi\right)(x)
$$

where $\phi \in\left\{\phi(t): \phi^{\prime}(t) \in L_{p, \omega}\right\}, \omega>0,0<\alpha<1$. 
Marchaud's ideas is applied in the study of another constructions, such as the Hadamard type derivative in [29]:

$$
\left(\mathcal{D}_{0+, \mu}^{(\alpha)} f\right)=x^{1-\mu} \frac{d}{d x} \frac{1}{\Gamma(1-\alpha)} \int_{0}^{x}\left(\log \frac{x}{t}\right)^{-\alpha} \frac{f(t) d t}{t^{1-\mu}} \quad(0<\alpha<1) .
$$

It was shown that, for any function $f \in X_{c}^{p}\left(\mathbb{R}_{+}\right)=\left\{g:\left(\int_{0}^{\infty}\left|t^{c} g(t)\right|^{p} \frac{d t}{t}\right)^{1 / p}<+\infty\right\}$, the following relation holds: $\left(\mathbf{D}_{0+, \mu}^{\alpha} f\right)(x)=\left(\mathcal{D}_{0+, \mu}^{(\alpha)} f\right), 0<\alpha<1$, where

$$
\begin{gathered}
\left(\mathbf{D}_{0+, \mu}^{\alpha} f\right)(x)=\frac{\alpha}{\Gamma(1-\alpha)} \int_{0}^{\infty} e^{-\mu t} \frac{f(x)-f\left(x e^{-t}\right)}{t^{1+\alpha}} d t+\mu^{\alpha} f(x)= \\
=\frac{\alpha}{\Gamma(1-\alpha)} \int_{0}^{x}\left(\frac{t}{x}\right)^{\mu}\left(\log \frac{x}{t}\right)^{-\alpha-1} \frac{f(x)-f(t)}{t} d t+\mu^{\alpha} f(x) .
\end{gathered}
$$

The difference between the Marchaud derivatives on semi-axes and the Liouville derivatives (e.g., for small values of parameter $\alpha, 0<\alpha<1$ )

$$
\left(\mathcal{D}_{+}^{\alpha} f\right)(x)=\frac{1}{\Gamma(1-\alpha)} \frac{d}{d x} \int_{-\infty}^{x} \frac{f(t) d t}{(x-t)^{\alpha}},\left(\mathcal{D}_{-}^{\alpha} f\right)(x)=-\frac{1}{\Gamma(1-\alpha)} \frac{d}{d x} \int_{-\infty}^{x} \frac{f(t) d t}{(x-t)^{\alpha}}
$$

is related to invertibility of fractional integral. Thus, the identity:

$$
\mathcal{D}_{ \pm}^{\alpha} I_{ \pm}^{\alpha} f \equiv f
$$

is valid only for $f \in L_{1}\left(\mathbb{R}^{1}\right)$, but the identity:

$$
\mathbb{D}_{ \pm}^{\alpha} I_{ \pm}^{\alpha} f \equiv f
$$

is valid for all $f \in L_{p}\left(\mathbb{R}^{1}\right), 1 \leq p<1 / \alpha$. Analogous results are valid for the Marchaud derivatives on a finite interval and the Riemann-Liouville fractional derivative: if $f=I_{a+}^{\alpha} \varphi, \varphi \in L_{1}(a, b)$, then the Marchaud derivatives on the interval $(a, b)$ and the Riemann-Liouville derivatives coincide almost everywhere (see [10], Theorem 13.1). In particular, these derivatives coincide (a.e.) for all functions $f$ absolutely continuous on $(a, b)$.

Since the Marchaud fractional derivative is defined via finite differences, then it follows that sufficient conditions on a function $f$ to have the Marchaud fractional derivative of any order $\leq \alpha$ is the convergence of the integral (see [8]):

$$
\int_{0} \frac{\omega_{n}(t) d t}{t^{\alpha+1}}
$$

where $\omega_{n}$ is the finite difference of $f$ of order $n=[\alpha+1]$. Vice versa, if such integral diverges for certain $\alpha>0$, then the function $f$ does not have a bounded fractional Marchaud derivative of any order greater than $\alpha$. One of the possible ways of using the Marchaud derivative in a discrete case is discussed in [28].

Marchaud fractional derivative has a form similar to Riesz fractional derivative (or hypersingular integral) and thus possesses multidimensional generalization (see [10], Section 26). One of the most prominent constructions for certain domains $\Omega \subseteq \mathbb{R}^{n}$ is given in [38]: 


$$
\left(\mathbb{D}_{\Omega}^{\alpha} f\right)(\mathbf{x})=c(\alpha)\left[a_{\Omega}(\mathbf{x}) f(\mathbf{x})+\int_{\Omega} \frac{f(\mathbf{x})-f(\mathbf{y})}{|\mathbf{x}-\mathbf{y}|^{n+\alpha}} d \mathbf{y}\right], \quad \mathbf{x} \in \Omega,
$$

where

$$
a_{\Omega}(\mathbf{x})=\int_{\mathbb{R}^{n} \backslash \Omega} \frac{d \mathbf{y}}{|\mathbf{x}-\mathbf{y}|^{n+\alpha}}, \quad c(\alpha)=\frac{2^{\alpha} \Gamma(1+\alpha / 2) \Gamma((n+\alpha) / 2) \sin (\pi \alpha) / 2}{\pi^{1+n / 2}} .
$$

\subsection{Short Biography of A.P. Marchaud}

André Paul Marchaud was born (Here, we follow the biographical paper [39].) in Saintes (Charente-Maritime) on 27 April 1887 in a religious family (Protestants). He received his primary education at Saintes and Paris, and then returned to secondary school in Saintes. At the age 17, he got ill with pleurisy. He obtained his Bachelor's degree in 1906, and then studied in College Chaptal preparing for entering École normal supérieure or École polytechnique. Marchaud studied at the École normal supérieure from 1909-1912, got his Master's degree in 1911. After graduating from École normal supérieure, he obtained an aggregation in mathematics in 1912. He was a teacher in mathematics at the high school in Montauban (from 1 October 1913 until 2 October 1919).

In 1914, Marchaud was mobilized as a second lieutenant of the 344 infantry regiment of Bordeaux. He was captured on 20 August 1914, and transferred to Germany. He was a prisoner for more than two years. In 1917, Marchaud became ill and was moved to Switzerland by the Red Cross. He returned to France on 6 July 1918.

In France, Marchaud continued his work as a teacher in mathematics at high schools (in Montauban and in Monpellier). In March, 1927, he defended a Doctor of Science thesis "On derivatives and differences of functions of real variables" (chairman of the committee Montel, examiners Denjoy and Chazy). In the period from October 1927 until October 1938, he occupied different positions at the mathematical faculty in Marseille. A.P. Marchaud was a rector of the Academy Clermont (1938-1944), Academy of Bordeaux (1944-1950) and a member of rectorate of the city University, Paris (1950-1957). He retired in August 1957 and passed away on 15 October 1973.

\section{Conclusions}

In this paper, we describe two approaches to the definition of fractional derivatives. The aim of both constructions is to define a fractional derivative that can be applied to a wide class of functions.

Letnikov's (1) and Marchaud's (3) derivatives are based on the use of differences. Thus, Letnikov studied the properties of differences of an arbitrary order and applied them to define his derivative. Marchaud proposed the regularization of the divergent integral by using finite differences of an integer order. The functions to which these constructions can be applied are not differentiable in general. One more common feature of these fractional derivatives is that both of them are started from the Riemann-Liouville fractional derivative, which needs to be simplified in order to be applied to a rather wide class of functions.

We also have to mention the following result on coincidence of Letnikov's and Marchaud's derivatives.

Theorem 2. (Theorem 20.4 [10]) Let $f \in L_{r}(\mathbb{R})$ for certain $1 \leq r<\infty$. Then, the following limits $\left.f_{ \pm}^{\alpha}\right)=\lim _{h \rightarrow+0,\left(L_{p}(\mathbb{R})\right)} \frac{\left(\Delta_{ \pm h}^{\alpha} f\right)(x)}{h^{\alpha}}$ and $\left(\mathbb{D}_{ \pm}^{\alpha} f\right)(x)=\lim _{\varepsilon \rightarrow+0,\left(L_{p}(\mathbb{R})\right)}\left(\mathbb{D}_{ \pm, \varepsilon}^{\alpha} f\right)(x)$ exist simultaneously and coincide (for the same choice of signs and $1<p<1 / \alpha$ ).

Letnikov's idea is briefly mentioned in the main paper by Marchaud in spite of the fact that Marchaud never read the paper by Letnikov or Grünwald. Since 50 years separate Letnikov's and Marchaud's proposals, we can see that Marchaud's paper already explored the results from the theory functions, the theory of sets and the theory of integration developed at the beginning of the 20th century by French and Russian schools.

In conclusion, we can say that both constructions are prominent and we suppose that many mathematicians would apply them in their work. 
Acknowledgments: The work has received funding from the People Programme (Marie Curie Actions) of the European Union's Seventh Framework Programme FP7/2007-2013/ under the REAGrant Agreement PIRSES-GA-2013-610547-TAMER and supported by the Belarusian Fund for Fundamental Scientific Research through the grant F17MS-002.

Author Contributions: The authors made equal contributions in the article.

Conflicts of Interest: The authors declare no conflict of interest.

\section{References}

1. Letnikov, A.V.; Chernykh, V.A. The Foundation of Fractional Calculus (with Applications to the Theory of Oil and Gas Production, Underground Hydrodynamics and Dynamics of Biological Systems); Neftegaz: Moscow, Russia, 2011. (In Russian)

2. Grünwald, A.K. Über “begrentze” Derivationen und deren Anwendung. Z. Angew. Math. Phys. 1867, 12, 441-480.

3. Liouville, J. Mémoire sur quelques questiones de géométrie et de mécanique, et nouveau gendre de calcul pour résoudre ces questiones. J. Ecole Roy. Polytéchn. 1832, 13, 1-69.

4. Liouville, J. Mémoire sur le calcul des différentielles a indices quelconques. J. Ecole Roy. Polytéchn. 1832, 13, 71-162.

5. Letnikov, A.V. Theory of differentiation of an arbitrary order. Mat. Sb. 1868, 3, 1-68. (In Russian)

6. Letnikov, A.V. An investigation related to the theory of integrals of the form $\int_{a}^{x}(x-u)^{p-1} f(u) d u$. Mat. Sb. 1874, 7, 5-205. (In Russian)

7. Riemann, B.; Weber, H . Gesammelte Mathematische Werke und Wissenschaflicher Nachlass; Teubner: Leipzig, Germany, 1876 .

8. Marchaud, A.P. Sur les dérivées et sur les différences des fonctions de variablr réelles. J. Math. Pure Appl. 1927, 6, 337-426. (In French)

9. Marchaud, A.P. Sur les Dérivées et sur les Différences des Fonctions de Variablr Réelles; Thésis de Entre-Deux-Guerres; Numdam, The French Digital Mathematics Library: Paris, France, 1965. (In French)

10. Samko, S.G.; Kilbas, A.A.; Marichev, O.I. Fractional Integrals and Derivatives: Theory and Applications; Breach Science Publishers: London, UK, 1993.

11. Baleanu, D.; Diethelm, K.; Scalas, E.; Trujillo, J.J. Fractional Calculus: Models and Numerical Methods, 2nd ed.; Series on Complexity, Nonlinearity and Chaos; World Scientific: Singapore, 2016.

12. Almeida, R.; Pooseh, S.; Torres D. Computational Methods in the Fractional Calculus of Variations; Imperial College Press and World Scientific: Singapore, 2015.

13. Uchaikin, V. Fractional Derivatives for Physicists and Engineers; Springer: Berlin, Germany; Higher Education Press: Beijing, China, 2013.

14. Atanackovic, T.; Philipović, S., Stanković, B., Zorica, D. Fractional Calculus with Applications in Mechanics: Vibrations and Diffusion Processes; Wiley: London, UK, 2014.

15. Gorenflo, R.; Kilbas, A.; Mainardi, F.; Rogosin, S. Mittag-Leffler Functions, Related Topics and Applications; Springer: Berlin/Heidelberg, Germany, 2014.

16. Podlubny, I. Fractional Differential Equations; Academic Press: New York, NY, USA, 1999.

17. Caponetto, R.; Dongola, G.; Fortuna, L.; Petráš, I. Fractional Order Systems: Modeling and Control Applications; World Scientific: Singapore, 2010.

18. Hilfer, R. (Ed.) Applications of Fractional Calculus in Physics; World Scientific: Singapore, 2000.

19. Mainardi, F. Fractional Calculus and Waves in Linear Viscoelasticity; Imperial College Press and World Scientific: Singapore, 2010.

20. Hille, E.; Tamarkin, J.D. On the theory of linear integral equations. Ann. Math. 1930, 31, 479-528.

21. Miller, K.S.; Ross, B. An Introduction to the Fractional Calculus and Fractional Differential Equations; John Wiley and Sons: New York, NY, USA, 1993.

22. Kilbas, A.A.; Srivastava, H.M.; Trujillo, J.J. Theory and Applications of Fractional Differential Equations; Elsevier: Amsterdam, The Netherlands, 2006.

23. Allen, M.; Caffarelli, L.; Vasseur, A. A parabolic problem with a fractional time derivative, Arch. Rat. Mech. Anal. 2016, 221, 603-630. 
24. Bergounioux, M.; Leaci, A.; Nardi, G.; Tomarelli, F. Fractional Sobolev spaces and functions of bounded variation of one variable. Frac. Calc. Appl. Anal. 2017, 20, 936-962.

25. Bucur, C.; Ferrari, F. An extesion problem for the fractional derivative defined by Marchaud. Frac. Calc. Appl. Anal. 2016, 19, 867-887.

26. West, B. J.; Bologna, M.; Grigolini, P. Physics of Fractal Operators; Springer: New York, NY, USA, 2003.

27. Yao, K.; Su, W.-Y.; Zhou, S.P. The fractional derivative of a fractal functions. Acta Math. Sinica. Engl. Ser. 2006, 22, 719-722.

28. Tarasova, V.V.; Tarasov, V.E. Exact discretization of economic accelerator and multiplier with memory. Fractal Fract. 2017, 1, 6 .

29. Kilbas, A.A.; Titioura, A.A. Nonlinear differential equations with Marchaud-Hadamard-type fractional derivative in the weighted spaces of summable functions. Math. Model. Anal. 2007, 12, 343-356.

30. Tenreiro Machado, J.; Kiryakova, V.; Mainardi, F. A poster about the old history of fractional calculus. Frac. Calc. Appl. Anal. 2010, 13, 447-454.

31. Nekrasov, P.A. General differentiation. Mat. Sb. 1888, 14, 45-168. (In Russian)

32. Butzer, P.L.; Westphal, U. An access to fractional differentiation via fractional difference quotients. Lect. Notes Math. 1975, 457, 116-145.

33. Bugrov, J.S. Fractional difference operators and classes of functions. Trudy Matematicheskogo Instituta imeni VA Steklova 1985, 172, 60-70. (In Russian)

34. Letnikov, A.V. On explanation of the main provisions of the theory of differentiation of arbitrary order. Mat. Sb. 1872, 6, 413-445. (In Russian)

35. Sonin, N.Y. On differentiation of arbitrary order. Mat. Sb. 1872, 6, 1-38. (In Russian)

36. Montel, P. Sure les polynomes d'approximation. Bull. Soc. Math. Fr. 1918, 46, 151-192.

37. De la Vallee Poussin, C.-J. Over differentie quotienten en differential quotienten. K. Ak. Wetensch. Amsterdam 1908, 17, 38-45.

38. Rafeiro, H.; Samko, S. On multidimensional analogue of Marchaud formula for fractional Riesz-type derivatives in domains in $\mathbb{R}^{n}$. Frac. Calc. Appl. Anal. 2005, 8, 393-401.

39. Condette, J.-F. Marchaud André Paul. In Les Recteurs d'académie en France de 1808 à 1940, Tom II; Dictionnaire biographique; Institut National de Recherche Pédagogique: Paris, France, 2006; pp. 272-273.

(C) 2017 by the authors. Licensee MDPI, Basel, Switzerland. This article is an open access article distributed under the terms and conditions of the Creative Commons Attribution (CC BY) license (http:/ / creativecommons.org/licenses/by/4.0/). 